\title{
Lobeline Docking on AChBP and Nicotinic Receptors: Discriminating Importance of the Pocket Geometry and of the Ligand Configuration
}

\author{
Claire Colas ${ }^{1}$, Xavier Brotel ${ }^{1}$, Nathalie Duclert-Savatier ${ }^{1}$, Michael Nilges ${ }^{1}$, Delphine Joseph ${ }^{2}$ and \\ Thérèse E. Malliavin*,1 \\ ${ }^{1}$ Unité de Bioinformatique Structurale, CNRS URA 2185, Institut Pasteur 25-28 rue du Dr Roux, F-75724, Paris Cedex \\ 15, France \\ ${ }^{2}$ BIOCiS UMR CNRS 8076, Faculté Chatenay-Malabry, Université Paris-Sud, France
}

Received May 31, 2011: Revised August 08, 2011: Accepted September 26, 2011

\begin{abstract}
Docking of lobeline, a partial agonist of nicotinic acetylcholine receptors (nAChRs), was investigated at once into crystallographic structures of acetylcholine binding proteins (AChBP) and into $\alpha 7$ and $\alpha 4 \beta 2 \mathrm{nAChRs} \mathrm{homology}$ models, and compared to behavior of full agonists, nicotine and epibatidine. The homology models were built using as templates the different pocket geometries established in crystallographic AChBP structures. Systematic cross-docking of each ligand into binding pockets of the two other ligands as well as its self-docking into its own pocket were performed in order to better understand the structural features determining the binding of these three ligands chosen for their molecular diversity. In AChBPs, epibatidine and nicotine display similar docking scores in their own pocket and in other ligands pockets: in particular, they also dock favorably into the lobeline pocket. In opposite, lobeline displays different features: it only binds favorably to its own pocket in AChBPs. Furthermore, the docking poses observed starting from lobeline stereoisomers support the importance of the intramolecular hydrogen bond between the alcohol function of the $\beta$-phenyl$\beta$-hydroxyethyl arm and the piperidinium proton for the lobeline binding to AChBP. For homology models, crossdockings are still discriminating and the specificity of lobeline for its binding pocket is conserved.
\end{abstract}

Keywords: AChBP, Lobeline, Docking, Diastereomer.

\section{INTRODUCTION}

Ligand-Gated Ion Channels (LGICs) are transmembrane channel proteins that are activated in response to neurotransmitters. Prototypic ligand-gated ion channels are nicotinic acetylcholine receptors (nAChRs), present in the central nervous system (CNS) and in the peripheral nervous system (PNS). These receptors are the subject of a renewed interest [1]. Indeed, nAChRs were shown to play a crucial role in smoking addiction [2], Alzheimer disease [3] and several neuropsychiatric disorders [4]. The chemical messengers binding to nAChRs can be classified into agonists which induce the channel opening, and antagonists which induce the channel closing.

Nicotinic acetylcholine receptors (nAChRs) are active as pentamers where the binding site is formed at the interface between two subunits in the $\mathrm{N}$-terminal extracellular domain [5]. This interface involves amino acid residues of two $\alpha$ subunits for homomeric pentamers (or of $\alpha$ and $\beta$ subunits in the case of heteromeric pentamers) [6]. The amino acid sequences constituting the binding site are arranged into six loops: loops $\mathrm{A}, \mathrm{B}$ and $\mathrm{C}$ belong to the principal component borne by the $\alpha$-subunit and loops D, E, and F to the $\alpha$ (or $\beta$ ) complementary one [7]. At least two agonists are required to open the pore by inducing a conformational change of all present subunits.

*Address correspondence to this author at the Unité de Bioinformatique Structurale, CNRS URA 2185, Institut Pasteur 25-28 rue du Dr Roux, F75724, Paris Cedex 15, France; Tel: +33 1406134 75;

Fax: +33 1456887 19; E-mail: terez@pasteur.fr
The nAChRs are allosteric membrane proteins and few information is available on their detailed atomic structure, despite enormous efforts for crystallization. In parallel, numerous high-resolution crystallographic structures of the Acetylcholine Binding Protein (AChBP), soluble homopentameric homologues to the extracellular domains of nAChRs, are available. These X-ray structures are in the apo [8] or in the holo form, cocrystallized with several agonists $[9,10]$ and antagonists [11-13], and reveal [14] atomic details of the interactions of these ligands with a pocket located at the subunits interface and capped by the loop C. Agonists bind to a small pocket limited by the closed loop C, whereas antagonists which are usually bulkier ligands, benefit from a larger binding pocket due to the loop $\mathrm{C}$ opening. Nevertheless, motions of other interface regions [15] can be involved in the discrimination between agonists and antagonists.

As no high-resolution X-ray nAChRs structures are up to now available, homology modeling approaches [16] were extensively used for molecular modeling and ligand docking studies. These homology models allowed to perform: molecular dynamics simulations [17-21], steered-molecular dynamics [22, 23] and ligand interaction analysis [24-27] Docking studies of nAChR ligands on different pocket geometries have been conducted [28-37], but, to our knowledge, none has been involving extensive docking of lobeline.

Lobeline is a natural alkaloid found in several Lobelia species. Lobeline has a particularly complex pharmacological profile [38], as it can be presented as an agonist [39] or an antagonist [40] of nicotine toward 
nAChRs or more as a nAChR partial agonist [41]. The isolated lobeline was also described to exhibit exceptional hydrogen-bond properties [42] which may be relevant for its biological activity. Furthermore, literature described that lobeline was able to epimerize in buffer solutions [43] as well as in polar organic solvents [44]: cis-(-)-lobeline is converted into trans-lobeline by isomerization of the phenacyl arm. This lobeline epimerization might also be responsible of its particular pharmacological profile. As a new mechanism of action of the partial agonists was recently [45] proposed, it is interesting to analyze how lobeline can be docked to AChBP structures and homology models of nAChR extra-cellular domains.

In this context, we study the interactions between $\mathrm{AChBP}$, and three evidenced nAChR ligands such as (-)nicotine, (+)-epibatidine and (-)-lobeline. (-)-Nicotine and (+)-epibatidine have been chosen as typical agonists for which the AChBP co-crystals were accessible. The study was also conducted on homology models of $(\alpha 7)_{2}$ and $\alpha 4 \beta 2$ dimers, as $\alpha 7$ and $\alpha 4 \beta 2$ nAChRs correspond to important pharmacological targets. Nicotine is an agonist displaying better affinity for $\alpha 4 \beta 2$ than for $\alpha 7$ nAChR subtype [46], whereas epibatidine is a much more potent agonist than nicotine, but displays no selectivity between $\alpha 7$ and $\alpha 4 \beta 2$ [47]. Nevertheless, a better affinity of epibatidine to $\alpha 4 \beta 2$ with respect to $\alpha 7$ was also reported [48, 49]. This discrepancy may arise from the use of nAChRs extracted from different organisms. Lobeline has been reported to be a better antagonist for human $\alpha 4 \beta 2 \mathrm{nAChRs}\left(\mathrm{K}_{\mathrm{i}}=4.7 \mathrm{nM}\right)$ [50] than for $\alpha 7 \mathrm{nAChRs}\left(\mathrm{K}_{\mathrm{i}}=6.26 \mu \mathrm{M}\right)$ [51] indicating a better affinity for $\alpha 4 \beta 2$ than for $\alpha 7 \mathrm{nAChR}$ subtypes.

The protein-ligand interaction was studied starting from the three protonated ligands to mimic physiological binding conditions, protonation occurring on the saturated heterocyclic basic nitrogen. Consider that the ligandreceptor interaction producing a biological activity is the consequence of the dynamic equilibrium between protonation and deprotonation of the tertiary ammonium in physiological conditions, the X-ray co-crystals of these two considered ligands in AChBP may not reveal the ligand orientation in the binding pocket observed in vivo. Thus, we decided to compare the docking behavior of the different diastereoisomers of lobeline and nicotine.

The different binding pocket geometries were compared by systematic cross-docking of each protonated ligand into the binding pockets of the other ligands. Cross-docking of these three ammonium ligands into AChBP structures or into homology models of $\alpha 7, \alpha 4$ and $\beta 2$ nAChR subunits, reveals conspicuous behaviors. In AChBP, the epibatidine and nicotine pockets bind both ligands, but, as expected, protonated lobeline binds favorably only into its own pocket [52]. Beside, two lobeline stereoisomers give the best docking solutions. In homology models, it transpires that the situation is more contrasted. Indeed, appropriate mutations of the sequence were necessary to display a docking of each ligands specific to its own pocket geometry, and to obtain scores in agreement with experimental specificity observed for epibatidine and nicotine to $\alpha 7$ and $\alpha 4 \beta 2 \mathrm{nAChR}$ subtypes. The mutation requirement reveals limitations in the reliability of the homology models. Nevertheless, the trends of binding pocket specificity, observed for lobeline in AChBP, are still valid for nAChR homology models.

\section{MATERIALS AND METHODS}

The following X-ray crystallographic structures of AChBP were used: 1UW6 [9] from Lymnaea stagnalis bound to (-)-nicotine, 2BYQ and 2BYS [53] from Aplysia californica bound to (+)-epibatidine and (-)-lobeline respectively. Chains $\mathrm{A}, \mathrm{B}$ and $\mathrm{E}$ were extracted from the PDB structures to perform docking studies. The docking on $\mathrm{X}$-ray crystallographic structures was done on the dimers $\mathrm{AB}$ in 1UW6 and 2BYS and on dimer EA in 2BYQ.

Sequences of subunits $\alpha 7, \alpha 4$ and $\beta 2$ of nAChRs (P36544, P43681, P17787 from Uniprot http://www. uniprot.org/) were aligned to the subunits of AChBP from Aplysia californica and Lymnaea stagnalis, using 3DCoffee [54]. The sequences alignments and X-ray crystallographic structures 1UW6, 1BYQ and 2BYS, were used to build homology models of subunits $\alpha 7, \alpha 4$ and $\beta 2$ using Modeller $9 v 6$ [16]. The subunits were then combined in order to obtain homology models of $(\alpha 7)_{2}$ and $\alpha 4 \beta 2$ dimers. The quality of homology models was checked using WHATIF 7.1 [55], and was found acceptable.

The three dimensional (3D) atomic coordinates of the protonated structures of (-)-nicotine and (+)-epibatidine were generated using Chem3D Ultra 9.0 (Cambridge Software copyright 1986-2004) and the more stable conformation was calculated using an MM2 energy-minimization. The 3D structure has been constructed starting from the crystallographic structures of ligands in AChBP. For protonated nicotine, both substituents are in anti configuration. The protonated lobeline stereoisomer contained in the structure 2BYS [53] was found to be the (+)-enantiomer of the natural alkaloid although the crystal had been prepared starting from (-)-lobeline hydrochloride ordered from Tocris Bioscience [53]. Thus, we decided to use protonated (-)-lobeline built using ChemDraw Ultra rather than its enantiomer present in 2BYS. Similarly to the nicotine building, the constructed 3D structure of lobeline mimics the crystallographic structure, in which the three substituents of the piperidine core are in syn configuration.

It is noteworthy that (-)-lobeline (Fig. 1a) is formed by a $\mathrm{N}$-methyl piperidine core, to which two arms are attached in positions 2 and 5: a phenyl 2-keto-ethyl and a phenyl 2(S)hydroxyethyl groups respectively. Lobeline as other ketopiperidine alkaloids is configurationnally unstable and easily undergoes epimerization [43, 44] of the phenacyl substituent. It has been shown that the rate of mutarotation of cislobeline affording a mixture of cis- $(2 R, 6 S, 10 S)$ and translobeline $(2 S, 6 S, 10 S)$ is increased in hydrophilic solvent such as physiological medium [56]. To better define the mutual influence between the lobeline epimerisation and its interaction with nAChRs, we decided to explore using docking approach which stereoisomer would present the best level of interaction with AChBP. The two diastereoisomers of (-)-lobeline (Fig. 1a- $(1 R, 2 R, 6 S, 10 S))^{1}$ corresponding to

\footnotetext{
${ }^{1}$ As, for the docking experiments, the stereogenic centre configurations were imposed by the scaffolds 3D construction, we adopted the R/S labeling for the stereogenic
} tertiary ammonium centre by analogy to asymmetric carbon rules. 
a.<smiles>C[N+](C)(CCC(=O)c1ccccc1)[C@H]1CC[C@@H]1C[C@@H](O)c1ccccc1</smiles>

c.

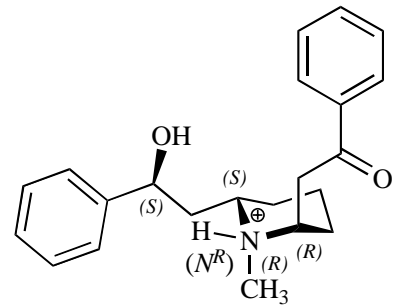

b.<smiles>CN(C1CC1)[N+](C)(C)CC[C@H](O)c1ccccc1</smiles>

d.

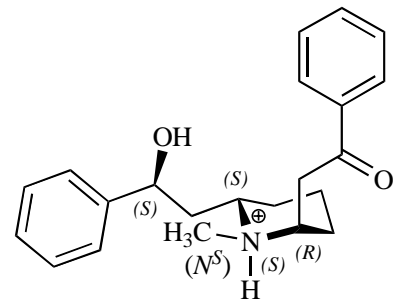

Fig. (1). Lobeline stereoisomers; a) Protonated axial $N$-methyl (-)-lobeline diastereomer ((1R)-(-)-lobeline), b) protonated equatorial $N$ methyl (-)-lobeline diastereomer $((1 S)-(-)$-lobeline), c) protonated axial $N$-methyl 2-epi-lobeline diastereomer $((1 R)$-2-epi-lobeline), d) protonated equatorial $\mathrm{N}$-methyl 2-epi-lobeline diastereomer ((1S)-2-epi-lobeline).

the inversion of the piperidinium nitrogen (Fig. 1b-(1S, $2 R$, $6 S, 10 S))$, and the epimerization of the phenyl 2-keto-ethyl arm (Fig. 1c- $(1 R, 2 S, 6 S, 10 S))$, as well as the diastereoisomer produced by the inversion of these two chiral centers (Fig. 1d-(1S, $2 S, 6 S, 10 S)$ ) were built using Chem3D Ultra. Similarly, the stereoisomer of (-)-nicotine (anti relative configuration of both pyrrolidinium substituents) corresponding to the inversion of pyrrolidinium nitrogen (syn relative configuration of both pyrrolidinium substituents) was also built using Chem3D Ultra: the two nicotine diastereoisomers are sketched in Fig. 2 (Figs. 2a: $(1 R, 2 S)$ and $\mathbf{2 b}:(1 S, 2 S))$.
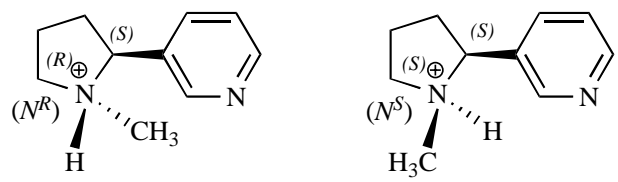

Fig. (2). Nicotine stereoisomers; a) (1R)-nicotine, b) (1S)-nicotine.

The docking of ligands to the proteins was performed using the script dock6grid-Lig of the software ICM [57]. The docking procedure was based on a Monte Carlo algorithm, allowing the exploration of the ligand conformations in the torsion angle space [58, 59]. The protein electrostatic potential was a distance-dependent potential with a grid size of $0.5 \AA$, and the van der Waals potential used with an interaction cutoff of $4.0 \AA$.

30 poses were calculated for each ligand docking. The score value is calculated for each ligand conformation using a linear combination of energetic terms as well as the number of ligand atoms: the weights of these terms have been determined by a training on a set of 23 receptors and 62 ligands [60]. The residues defining the active pockets for the different $\mathrm{nAChRs}$ and AChBPs are given in Table $\mathbf{1}$.

\section{RESULTS}

\subsection{Cross-Docking on X-Ray Crystallographic Struct- ures of AChBP}

Cross-dockings of (-)-nicotine, (+)-epibatidine and (-)lobeline natural stereoisomers were performed, as described in introduction, on the crystallographic structures of the AChBP bound to the other ligands. Cross-dockings were

Table 1. Residues Numbers Defining the Docking Pockets

\begin{tabular}{|c|c|}
\hline pdb ID & pocket residues \\
\hline 2BYQ & W147-D155; V185-I196; E888-W892; S935-V941; M948-Q953 \\
\hline$\alpha 4 \beta 2-1 \mathrm{UW} 6$ & W147-D155;T185-P196; V262-Q266;V310-A316; I324-P328 \\
\hline$\alpha 4 \beta 2-2 B Y Q$ & W147-D155; T185-P196; V883-G887; V931-A937; I945-P949 \\
\hline$\alpha 7-2 B Y Q$ & G147-S155; E185-P196; W883-S887; T931-V936; Q945-L947 \\
\hline$\alpha 7-2 B Y S$ & G147-S155; E185-P196; W883-S887; T931-V936; Q945-L947 \\
\hline
\end{tabular}

The three X-ray crystallographic structures are designed by their PDB entries. The homology models are designed by the nAChR subunit name followed by the PDB entry of the model template. 


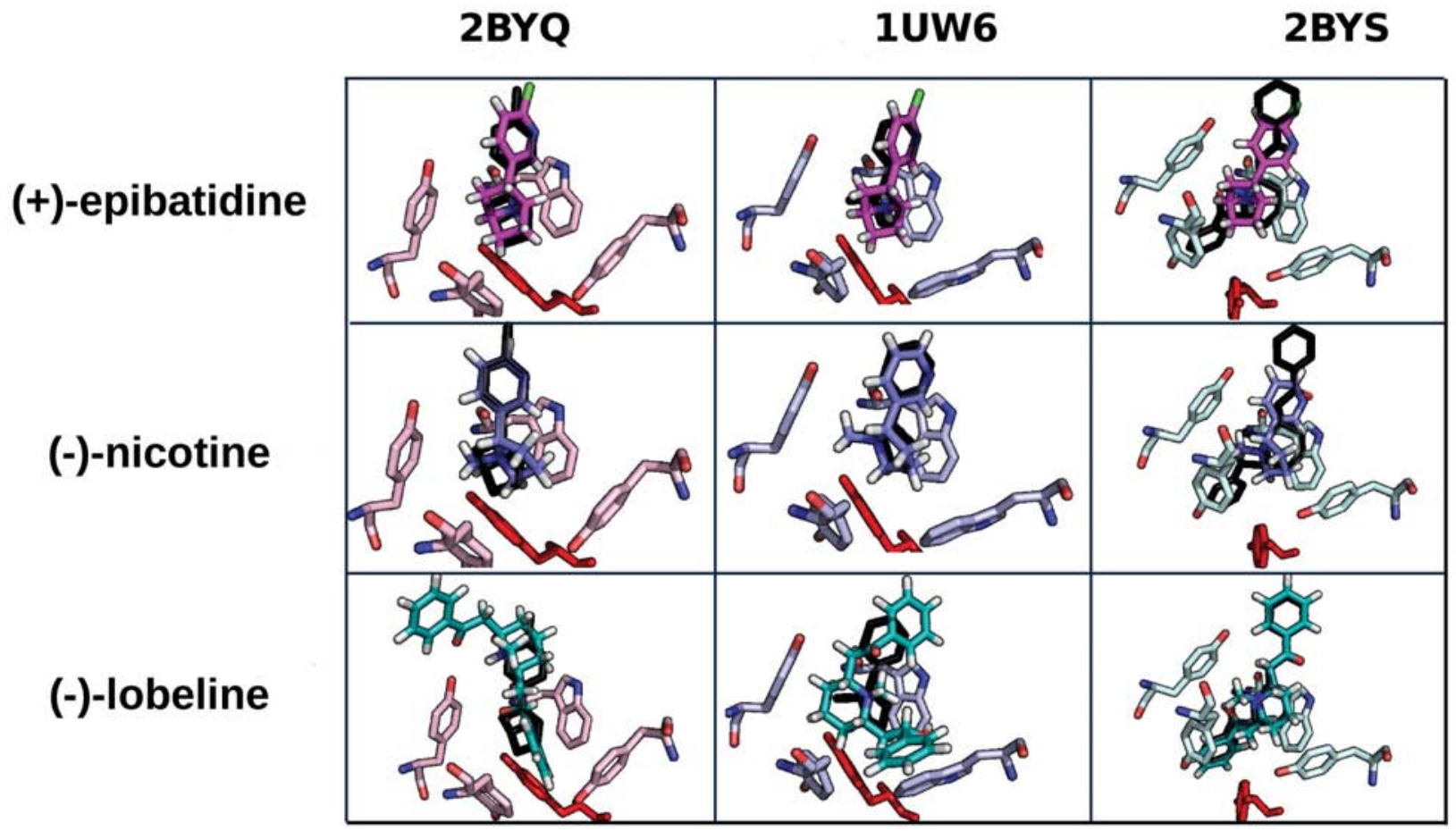

Fig. (3). Cross-docking of protonated (+)-epibatidine, (-)-nicotine and (-)-lobeline in 2BYQ, 1 UW6 and 2BYS. Proteins residues and ligands are represented in sticks: protein residue in atom color, and ligands in magenta (epibatidine), blue (nicotine) and cyan (lobeline). Ligands crystallographic poses are represented in black. In all proteins, Y91 is colored in red.

compared to self-dockings, which were realized by docking each ligand into the pocket, observed in the crystallographic structure bound to the same ligand. The best docking poses are chosen as the ones minimizing the coordinate RMSD of the ligand to its crystallographic pose.

The natural nicotine, epibatidine and lobeline stereoisomers were docked in the pockets of the crystallographic structures: 1UW6 (bound to nicotine), 2BYQ (bound to epibatidine), 2BYS (bound to lobeline). Cross-docking and self-docking poses of epibatidine (Fig. 3a,b,c) and nicotine (Fig. 3d,e,f) display scores in the range of -19.9 to -15.0 (Table 2 ). In all cases, the ligands establish favorable interactions with the pockets, as the self-docking and cross-docking scores are of the same order of value. The self-docking scores are nevertheless slightly better, and this improvement is reflected in the docking poses. Indeed, the best poses of nicotine obtained in 2BYQ superimposed well to the crystallographic pose of nicotine in 1UW6 (Fig. 3d), with coordinate RMSD value of $0.79 \AA$ (Table 2). But, epibatidine docking pose in 1UW6 (Fig. 3b) displays a worse superposition to the epibatidine crystallographic pose in 2BYQ, with a coordinate RMSD of $1.74 \AA$.

In the lobeline pocket (2BYS), nicotine displays a larger coordinate RMSD (1.74 ̊) to its crystallographic pose in 1UW6, at the contrary of epibatidine $(0.58 \AA)$. The bulkier shape of epibatidine with respect to nicotine supports the different tendencies observed for the two ligands, and the preference of epibatidine for the lobeline larger pocket.

Cross-docking of lobeline in 2BYQ and 1UW6 could not reproduce the crystallographic poses, as Tyr 91 is oriented differently in 2BYQ and 1UW6 compared to 2BYS (Fig. 3) and induces unfavorable steric hindrance to lobeline docking into the pocket [52]. Because of this conflicting interaction the ICM scores obtained for the lobeline docking in 2BYQ and 1UW6 are +13.5 and +31.4 respectively, and the coordinate RMSD between lobeline docking and crystallographic poses are larger than $5 \AA$ (Table 2).

The cross-dockings of nicotine and epibatidine respectively on $2 \mathrm{BYQ}, 2 \mathrm{BYS}$ and $1 \mathrm{UW} 6,2 \mathrm{BYS}$, produce

Table 2. Results of the Ligands Docking on the X-ray Crystallographic Structures 2BYQ, 1UW6 and 2BYS

\begin{tabular}{|c|c|c|c|c|c|c|c|c|c|}
\hline \multirow[t]{2}{*}{ AChBP } & \multicolumn{3}{|c|}{ 2BYQ } & \multicolumn{3}{|c|}{ 1UW6 } & \multicolumn{3}{|c|}{ 2BYS } \\
\hline & ICM Score & Pose rank & $\begin{array}{l}\text { Coordinate } \\
\text { RMSD (Å) }\end{array}$ & ICM Score & Pose rank & $\begin{array}{l}\text { Coordinate } \\
\text { RMSD (Å) }\end{array}$ & ICM Score & Pose rank & $\begin{array}{l}\text { Coordinate } \\
\text { RMSD (A) }\end{array}$ \\
\hline (+)-epibatidine & -15.4 & 3 & 0.64 & -15.0 & 2 & 1.74 & -17.9 & 2 & 0.58 \\
\hline (-)-nicotine & -15.3 & 2 & 0.79 & -19.9 & 2 & 0.41 & -17.7 & 1 & 1.57 \\
\hline (-)-lobeline & 13.5 & 25 & 5.6 & 31.4 & 22 & 6.1 & -34.9 & 1 & 0.48 \\
\hline
\end{tabular}

For each ligand and each pocket, the ICM score, the pose rank and the coordinate RMSD ( $\AA$ ) with respect to the crystallographic pose, are given. For each ligand, the pose was chosen as the closest to the crystallographic one when the coordinate RMSD could be calculated and otherwise as the first pose oriented as like as the (reference) ligand itself. 
conformations close to the crystallographic poses and displaying favorable docking scores. On the contrary, lobeline produces satisfactory docking poses only in the 2BYS pocket geometry, because of dissimilar orientations of Tyr91 in the different AChBP structures [52]. The geometry of the lobeline pocket seems thus to be exactly fitted to its ligand. This close fitting may be related to the complex pharmacological profile of this compound, which was described as an agonist [39], an antagonist [40] or a partial agonist [41] ligand.

\subsection{Effect of the Nicotine and Lobeline Configuration on the Docking Poses in AChBP}

The two stereoisomers (Fig. 2) built for nicotine, (1S)-(-)nicotine and $(1 R)-(-)$-nicotine, were docked on the structure 1UW6. A less favorable score is obtained for $(1 R)-(-)-$ nicotine and this stereoisomer is also more shifted from the crystallographic pose than (1S)-(-)-nicotine. The orientation of the methyl group attached to the pyrrolidinium nitrogen, influences thus the nicotine binding. These results confirm that the ligand orientation in the $\mathrm{nAChR}$ binding pocket and consequently, the favorable protein-ligand interactions are conditioned by the $\pi$-cation interaction between the ammonium ligand cation and the $\pi$-box from the highly conserved principal binding site component.

As the structure 2BYS does not contain the natural (-)lobeline stereoisomer [53], which is supposed to bind to AChBP, the docking of this ligand into the 2BYS binding pocket will be used to predict the (-)-lobeline pose.

The protonated (-)-lobeline (Fig. 1a, (1R)-(-)-lobeline) is composed of a $N$-methyl piperidine ring in a chair conformation, to which are attached the phenyl-2-keto-ethyl and the phenyl-2(S)-hydroxyethyl substituents in positions 2 and 6 respectively [61]. In solution at physiological $\mathrm{pH}$, the additional ammonium stereogenic centre allowed the formation of two potential diastereomers: the crystallographic structure of protonated lobeline in AChBP shows a syn-relation between the two substituting arms and the $N$-methyl group which occupies an axial position [8]. The nitrogen inversion generates an additional diastereoisomer in which the $N$-methyl group is in equatorial position (Fig. 1b, (1S)-(-)-lobeline) (see Note 1 in "Crossdocking on X-ray crystallographic structures of AChBP"). Moreover, the characterized isomerization of the piperidine
C-2 carbon atom bearing the phenacyl arm can generate two additional diastereomers: the C-2 epimer of the crystal structure (Fig. 1c, (1R)-2-epi-lobeline) and respectively its equatorial $N$-methyl diastereoisomer (Fig. 1d, (1S)-2-epilobeline). In summary, both nitrogen and C-2 atoms could be inverted on the piperidine ring. Due to the results of nicotine diastereomers docking associated with the complex pharmacological profile of lobeline, we decided to explore the docking of the four possible stereoisomers of (-)-lobeline to the 2BYS pocket.

In order to check the geometry of the built enantiomers, the lobeline diastereoisomers were compared to the crystallographic of the neutral isolated lobeline [42], and the crystallographic structure was found equivalent to the (-)lobeline. For each diastereoisomer, a set of docking poses was obtained using ICM, and the pose best fitting to the electronic density of 2BYS was chosen as the best docking pose. The ICM scores obtained for the best docking poses of the lobeline stereoisomers (Table 3) are remarkably better for the $(1 R)-(-)$-lobeline (Fig. 1a) and (1R)-2-epi-lobeline (Fig. 1c) than for the two other stereoisomers

A recent crystallographic and computational analysis [42] of the neutral (-)-lobeline has put in evidence the equilibrium of lobeline between two conformations, characterized by the formation or the disruption of an intramolecular hydrogen bond between the alcohol function of the phenylhydroxyethyl arm and the ammonium proton. The establishment of this hydrogen bond is thought to be mandatory for the interaction of lobeline with nAChRs [62]. In the present work, among the poses obtained for each diastereoisomer (Fig. 4), the distribution of distances between the oxygen atom of the hydroxyl function and the ammonium proton, are shifted to values smaller than $2.5 \AA$, for $(1 R)-(-)$-lobeline and (1R)-2-epi-lobeline. Indeed, $33.3 \%$ (respectively $38.7 \%$ ) of the docking poses obtained from the docking of $(1 R)-(-)$-lobeline (respectively (1R)-2-epilobeline) display an intramolecular hydrogen bond. On the other hand, the percentages of hydrogen bond formation are smaller $(19.3 \%$ and $0 \%)$ for the respective inverted nitrogen diastereoisomers.

The analysis performed on the intramolecular hydrogen bond and the ICM scores support the $(1 R)-(-)-$ lobeline and the $(1 R)$-2-epi-lobeline as the most probable stereoisomers present in the active site of $2 \mathrm{BYS}$. This result suggests that

Table 3. ICM scores from the docking of the different stereoisomers on the X-ray crystallographic AChBP structures. The nicotine stereoisomers are docked on 1UW6, and the lobeline stereoisomers are docked on 2BYS

\begin{tabular}{|c|c|c|c|}
\hline stereoisomers & ICM conformation rank & ICM score & Coordinate RMSD $(\mathbf{A})$ \\
\hline \hline$(1 S)-(-)-$ nicotine & 2 & -19.9 & 0.41 \\
\hline$(1 R)-(-)-$-nicotine & 2 & -13.7 & 0.87 \\
\hline$(1 R)-(-)-$ lobeline $^{\mathrm{a}}$ & 1 & -34.9 & 0.48 \\
\hline$(1 S)-(-)-$ lobeline $^{\mathrm{b}}$ & 4 & -18.8 & 0.88 \\
\hline$(1 R)-2-$ epi-lobeline $^{\mathrm{c}}$ & 1 & -29.9 & 0.63 \\
\hline$(1 S)-2-$ epi-lobeline $^{\mathrm{d}}$ & 1 & -18.1 & 1.18 \\
\hline
\end{tabular}

${ }^{\mathrm{a}}(1 \mathrm{R}, 2 \mathrm{R}, 6 \mathrm{~S})-6-\left[(2 \mathrm{~S})-H y d r o x y-2-\right.$ phenylethyl]-1-methyl-2-(2-oxo-2-phenylethyl)piperidinium; ${ }^{\mathrm{b}}$ (1S,2R,6S)-6-[(2S)-Hydroxy-2-phenylethyl]-1-methyl-2-(2-oxo-2-phenylethyl)piperidinium; $\quad$ (1R,2S,6S)-6-[(2S)-Hydroxy-2-phenylethyl]-1-methyl-2-(2-oxo-2-phenylethyl)piperidinium; d (1S,2S,6S)-6-[(2S)-Hydroxy-2-phenylethyl]-1-methyl-2-(2-oxo-2-phenylethyl)piperidinium 

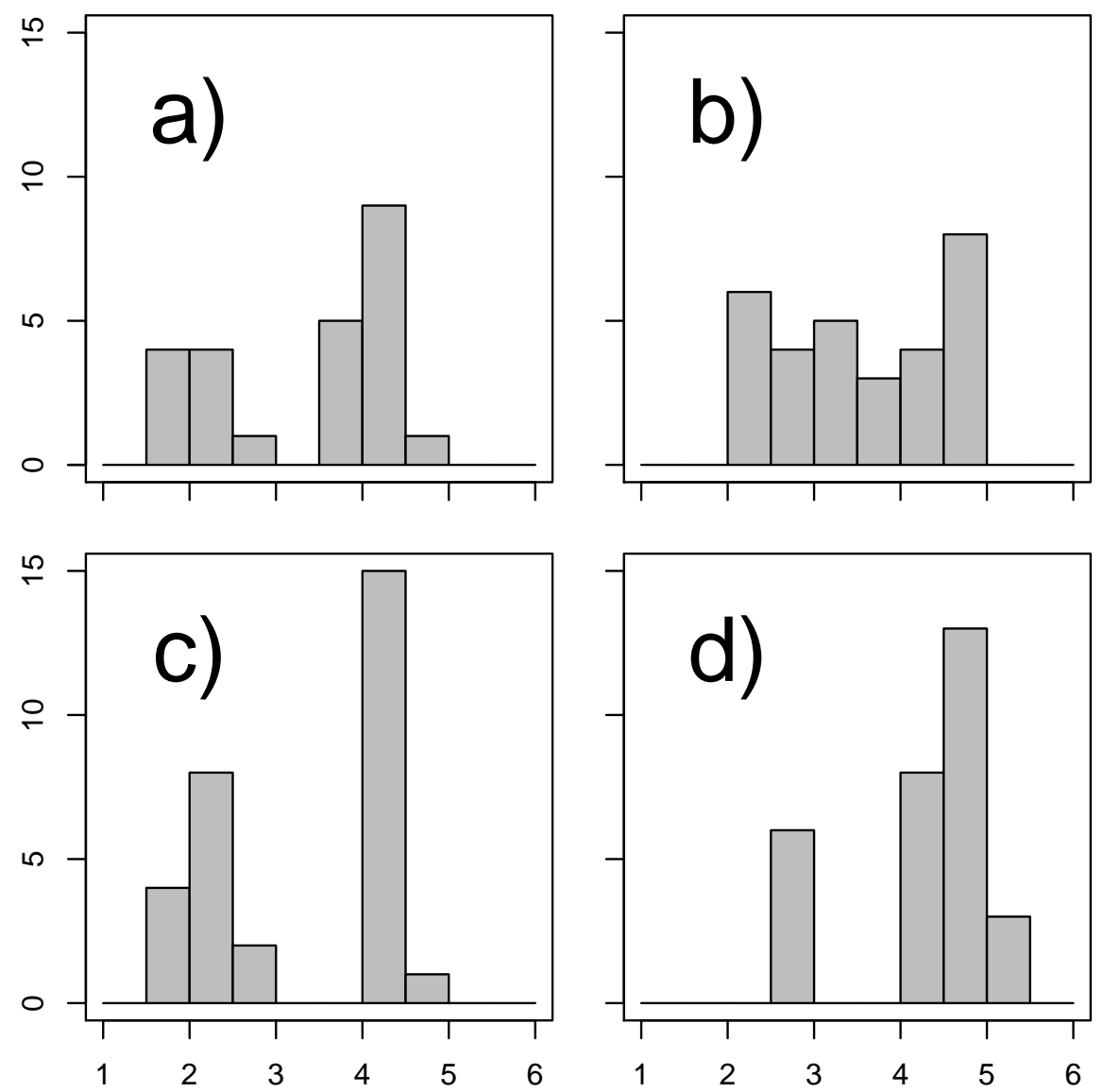

Fig. (4). Distribution of the distance $(\AA)$ between the alcohol function of the hydroxyethyl arm and the piperi- dinium proton in the different protonated lobeline stereoisomers. a) $(1 R)-(-)$-lobeline, b) (1S)-(-)-lobeline, c) (1R)-2-epi-lobeline, d) (1S)-2-epi-lobeline.

the orientation of the methyl group attached to the piperidine ammonium, determines the binding mode of lobeline: its orientation in the same direction as the hydroxyl group favors the formation of the intramolecular hydrogen bond, and support the importance [62] of this hydrogen bond for the interaction between lobeline and nAChRs.

\subsection{Docking on Homology Models}

The homology models built for $\alpha 7, \alpha 4$ and $\beta 2$ subunits were combined to produce dimers $(\alpha 7)_{2}$ and $\alpha 4 \beta 2$ corresponding to the binding sites of the nicotinic receptors the most often encountered in the Central Nervous System (CNS). The three crystallographic protonated ligands, (-)nicotine, (+)-epibatidine and (-)-lobeline were docked to all homology models, in order to investigate which is the influence of the template structure to the docking results (Table 4).

The docking on $\alpha 7$ homology models (Table 4a) displays unfavorable scores for all ligands in 2BYQ, for lobeline in 1UW6 and 2BYS, and for nicotine in 2BYS revealing that the electrostatic energy is repulsive. The residues R186 and K192 (both located in the C-loop) were picked up by looking for positively charged residues in the vicinity of the binding pocket. The repulsive effect of these two positively charged residues did not appear in AChBPs, as the corresponding residues in this protein are neutral (Q186 and P192).
Replacing the $\alpha 7$ sequence by the double-mutant R186QK192P, improved the scores (Table 4b). In the mutants, nicotine and epibatidine obtained favorable scores for selfdocking and cross-docking, similarly to the observations made on AChBP (Table 2). The effect of the electrostatic repulsion on the ligand docking should be put in relation with the study of Sgrignani et al. [63] where the importance of the ligand polarisability by the receptor is put in evidence.

Lobeline displays another tendency: the docking procedure found a lobeline pose with favorable score in 1UW6 pocket geometry, but this pose displays a large coordinate deviation from the lobeline crystallographic pose $(11.35 \AA)$. On the other hand, the only slightly favorable pose observed for lobeline in the $2 \mathrm{BYS}$ pocket geometry has a less favorable score (-2.5), but a closer RMSD to the crystallographic pose $(1.65 \AA)$. Also, the intramolecular hydrogen bond between the alcohol function of the $\beta$-phenyl$\beta$-hydroxyethyl arm and the piperidinium proton is established with a distance of 2.4 A. A general tendency of the docking on $(\alpha 7)_{2}$ models is that the coordinate RMSD from crystallographic poses increases in homology models with respect to AChBP.

The $\alpha 4 \beta 2$ dimers display a quite different behavior than the $(\alpha 7)_{2}$ dimers (Table 4a). Docking is quite discriminant for lobeline, a favorable score being obtained only for the 2BYS pocket geometry. Furthermore, the cross-dockings of epibatidine and nicotine become also discriminative. 
Table 4. ICM Scores (ranks) from the Docking of the Different Natural Ligands on the Homology Models of $\alpha 7$ and $\alpha 4 \beta 2$ Produced Using Different AChBPs Templates

\begin{tabular}{|c|c|c|c|c|c|c|c|c|c|}
\hline \multirow[t]{2}{*}{$\alpha 7$ template } & \multicolumn{3}{|c|}{ 2BYQ } & \multicolumn{3}{|c|}{ 1UW6 } & \multicolumn{3}{|c|}{ 2BYS } \\
\hline & score & rank & $\begin{array}{c}\text { Coord. } \\
\text { RMSD(Å) }\end{array}$ & score & rank & $\begin{array}{c}\text { Coord. } \\
\text { RMSD(Å) }\end{array}$ & score & rank & $\begin{array}{c}\text { Coord. } \\
\text { RMSD(Å) }\end{array}$ \\
\hline$(+)$-epibatidine ${ }^{a}$ & 0.4 & 7 & 0.61 & -0.4 & 6 & 1.14 & -0.3 & 6 & 1.29 \\
\hline$(+)$-epibatidine ${ }^{b}$ & -6.4 & 6 & 1.05 & -3.2 & 3 & 1.01 & 4.9 & 19 & 1.16 \\
\hline$(-)-$ nicotine ${ }^{a}$ & 4.0 & 7 & 1.31 & -2.3 & 7 & 0.48 & 9.6 & 16 & 1.11 \\
\hline$(-)-$ nicotine ${ }^{b}$ & -4.7 & 3 & 0.69 & -6.1 & 9 & 0.77 & 0.6 & 10 & 2.54 \\
\hline$(-)-$ lobeline $\mathrm{a}^{\mathrm{a}}$ & 20.4 & 11 & 7.21 & 15.2 & 24 & 7.6 & 1.9 & 2 & 1.90 \\
\hline$(-)$-lobeline ${ }^{b}$ & 30.9 & 30 & 4.14 & -6.3 & 6 & 11.35 & -2.5 & 4 & 1.65 \\
\hline \multirow[t]{2}{*}{$\alpha 4 \beta 2$ template } & \multicolumn{3}{|c|}{ 2BYQ } & \multicolumn{3}{|c|}{ 1UW6 } & \multicolumn{3}{|c|}{$2 B Y S$} \\
\hline & score & rank & $\begin{array}{c}\text { Coord. } \\
\text { RMSD(Å) }\end{array}$ & score & rank & $\begin{array}{c}\text { Coord. } \\
\text { RMSD(Å) }\end{array}$ & score & rank & $\begin{array}{c}\text { Coord. } \\
\text { RMSD(Å) }\end{array}$ \\
\hline (+)-epibatidine ${ }^{\mathrm{a}}$ & $\begin{array}{l}-7.8 \\
\end{array}$ & 1 & 0.76 & 7.9 & $\overline{9} 9$ & 1.27 & -1.35 & 10 & 1.80 \\
\hline$(-)-$ nicotine ${ }^{a}$ & -2.6 & 8 & 1.57 & -9.8 & 2 & 0.54 & -6.5 & 2 & 1.31 \\
\hline$(-)-$ lobeline $\mathrm{e}^{\mathrm{a}}$ & 19.6 & 23 & 6.54 & 36.3 & 30 & 6.54 & -10.6 & 1 & $0.77\left(2.4^{\mathrm{d}}\right)$ \\
\hline
\end{tabular}

adocking on the homology model. b docking on the homology model and double mutant R186Q-K192P.

Dockings of epibatidine and nicotine give similar scores whatever is the pocket geometry. Docking scores are worse with respect to the observations made in AChBP (Table 2).

Concerning the relative affinity of nicotine and epibatidine for $\alpha 7$ and $\alpha 4 \beta 2$ nAChR subtypes, the best scores observed for epibatidine on homology models, are -6.4 on $(\alpha 7)_{2}$, and -7.8 on $\alpha 4 \beta 2$ : these scores are in agreement with the lack of experimental specificity observed for epibatidine [47]. On the other hand, scores of -6.1 and -9.8 are observed for nicotine on $(\alpha 7)_{2}$ and $\alpha 4 \beta 2$ respectively, which agrees with the better experimental affinity of nicotine toward $\alpha 4 \beta 2$ nAChR subtype [46].

\section{DISCUSSION-CONCLUSION}

The (-)-lobeline was docked to different AChBP X-ray crystallographic structures as well as to nAChRs homologous models, and compared to the docking of two agonists of nAChRs ((-)-nicotine and (+)-epibatidine). Not surprisingly, the present study put in evidence that, depending on the agonist size and properties, exact geometry of the pocket sidechains, observed in X-ray crystallographic structures, can be determinant for finding a docking pose close to the crystallographic one. Different features are observed depending on the ligand, nicotine and epibatidine can dock favorably to different pocket shapes while it is not the case for lobeline.

Epibatidine and nicotine, which display similar sizes and binding modes ( $\pi$-cation interaction), display similar docking scores during self-docking and cross-docking, and are also able to dock favorably in the lobeline (2BYS) pocket. But, on the other hand, lobeline displays quite different docking behavior: it can dock with satisfying scores only on the 2BYS pocket. Lobeline is slightly larger than the structurally constrained nicotine and epibatidine and is a more elongated molecule due to the presence of its two flexible arms substituting the piperidine $\mathrm{C}-2$ and $\mathrm{C}-6$. Moreover, the binding of lobeline to AChBP involves stacking interactions with W147, van der Waals contacts with R79, M116, and I118, Y93, K143, G145, W147, and D197, and hydrogen bonds with the W47 indole nitrogen, S146 and W147 carbonyl oxygens: these interactions are quite different from the $\pi$-cation interaction observed in nicotine and epibatidine, and may be more discriminant.

Furthermore, lobeline diastereoisomers display different propensities to dock into the 2BYS pocket. Docking poses observed for lobeline diastereoisomers suggest that the orientation of the methyl group attached to the piperidine ammonium, determines the binding mode of lobeline. The agreement of these poses with experimental observation known on isolated and on AChBP-binding lobeline, supports the importance of an intramolecular hydrogen bond between the alcohol function of the phenylhydroxyethyl arm and the ammonium proton, for the lobeline binding to AChBP. The present docking work agrees with the conclusions obtained on the isolated lobeline by Locati et al. [42] concerning the importance of hydrogen bond formation for the lobeline affinity to nAChR. In the same trend than for lobeline, the nicotine docking is influenced by the orientation of the methyl group attached to the pyrrolidinium nitrogen.

Finally, the ligand docking behavior was explored on homology models of $(\alpha 7)_{2}$ and $\alpha 4 \beta 2$ dimers. The $\alpha 7$ subunit displays much more difficulties than the $\alpha 4$ subunit to reproduce results observed on $\mathrm{AChBP}$, as, for instance, mutations in the $\alpha 7$ sequence were necessary to obtain favorable scores. The sequence variations among the nAChRs subunits may thus induce a reorganization of the protein-ligand interactions and consequently of the ligand position in the pocket. Otherwise, the use of homology models makes cross-docking more discriminant. Also, the 
specificity of the lobeline for its own pocket geometry is conserved.

The present docking study intends to be a preliminary analysis of the lobeline docking behavior with respect to other agonists, in the perspective to mimic the lobeline scaffold for designing new potential partial agonists targeting selectively $\alpha 4 \beta 2 \mathrm{nAChR}$ subtypes for the development of new pharmacotherapies to treat addiction.

The outcome of this analysis gives indications on the design strategy. First, as the lobeline binding pocket is very specific, its shape should be used during any predictive docking study of lobeline analogues. Second, new lobelinelike compound design should favor a compact lobeline-type conformation by the formation of an intramolecular hydrogen bond between an alcohol function of the $\beta$-phenyl$\beta$-hydroxyethyl arm and the piperidinium proton.

\section{ACKNOWLEDGMENTS}

Dr. J. Graton is gratefully acknowledged for the gift of the YIGQOV file from the Cambridge crystallographic database, representing the structure of the isolated neutral lobeline. CNRS, Institut Pasteur and Université Paris-Sud are thanked for funding. The authors are grateful to the French Ministry of Superior Education and Research for the postgraduate fellowship of Claire Colas. Claire Colas thanks the Pasteur-Weizmann foundation for a three-month financial support for young researchers.

\section{CONFLICT OF INTEREST}

The authors have reported no conflict of interest.

\section{REFERENCES}

[1] Taly, A.; Corringer, P.; Guedin, D.; Lestage, P.; Changeux, J. Nicotinic receptors: allosteric transitions and therapeutic targets in the nervous system. Nat. Rev. Drug Discov., 2009, 8, 733-750.

[2] Foulds, J. The neurobiological basis for partial agonist treatment of nicotine dependence: varenicline. Int. J. Clin. Pract., 2006, 60, 571-576.

[3] Buckingham, S.; Jones, A.; Brown, L.; Sattelle, D. Nicotinic acetylcholine receptor signalling: roles in Alzheimer's disease and amyliod neuroprotection. Pharmacological. Rev., 2009, 61, 39-61.

[4] Newhouse, P.; Singh, A.; Potter, A. Nicotine and nicotinic receptor involvement in neuropsychiatric disor- ders. Curr. Top. Med. Chem., 2004, 4, 267-282.

[5] Miyazawa, A.; Fujiyoshi, Y.; Stowell M.; Unwin, N. Nicotinic acetylcholine receptor at $4.6 \mathrm{~A}$ resolution: trans- verse tunnels in the channel wall. J. Mol. Biol., 1999, 288, 765-786.

[6] Romanelli, M.; Gratteri, P.; Guandalini, L.; Martini, E.; Bonaccini, C.; Gualtieri, F. Central nicotinic recep- tors: structure, function, ligands, and therapeutic potential. ChemMedChem, 2007, 2, 746767.

[7] Unwin, N. Refined structure of the nicotinic acetylcholine receptor at 4 Å resolution. J. Mol. Biol., 2005, 346, 967-989.

[8] Brejc, K.; van Dijk, W.; Klaassen, R.; Schuurmans, M.; van Der Oost, J.; Smit, A.; Sixma, T. Crystal structure of an ACh-binding protein reveals the ligand-binding domain of nicotinic receptors. Nature, 2001, 411, 269-276.

[9] Celie, P.; van Rossum-Fikkert, S.; van Dijk, W.; Brejc, K.; Smit, A.; Sixma, K. Nicotine and carbamylcholine binding to nicotinic acetylcholine receptors as studied in AChBP crystal structures. Neuron, 2004, 41, 907-914.

[10] Hibbs, R.; Sulzenbacher, G.; Shi, J.; Talley, T.; Conrod, S.; Kem, W.; Taylor, P.; Marchot, P.; Bourne, Y. Structural determinants for interaction of partial agonists with acetylcholine binding protein and neuronal alpha7 nicotinic acetylcholine receptor. EMBO J. 2009, 28, 3040-3051.

[11] Celie, P.; Kasheverov, I.; Mordvintsev, D.; Hogg, R.; van Nierop, P.; van Elk, R.; van Rossum-Fikkert, S.; Zhmak, M.; Bertrand, D.; Tsetlin, V.; Sixma, T.; Smit, A. Crystal structure of nicotinic acetylcholine receptor homolog AChBP in complex with an alphaconotoxin PnIA variant. Nat. Struct. Biol., 2005, 12, 582-588.

[12] Bourne, Y.; Hansen, S.; Sulzenbacher, G.; Talley, T.; Huxford, T.; Taylor, P.; Marchot, P. Structural compari- son of three crystalline complexes of a peptidic toxin with a synaptic acetylcholine recognition protein. J. Mol. Neurosci., 2006, 30, 103-104.

[13] Ulens, C.; Hogg, R.; Celie, P.; Bertrand, D.; Tsetlin, V.; Smit, A.; Sixma, T. Structural determinants of selective alpha-conotoxin binding to a nicotinic acetylcholine receptor homolog AChBP. Proc. Natl. Acad. Sci. USA, 2006, 103, 3615-3620.

[14] Hansen, S.; Sulzenbacher, G.; Huxford, T.; Marchot, P.; Bourne, Y.; Taylor, P. Structural characterization of agonist and antagonistbound acetylcholine-binding protein from Aplysia californica. $J$. Mol. Neurosci., 2006, 30, 101-102.

[15] Hibbs, R.; Radic, Z.; Taylor, P.; Johnson, D. Influence of agonists and antagonists on the segmental motion of residues near the agonist binding pocket of the acetylcholine-binding protein. J. Biol. Chem., 2006, 281, 39708-39718.

[16] Sali, A.; Blundell, T. Comparative protein modelling by satisfaction of spatial restraints. J. Mol. Biol., 1993, 234, 779-815.

[17] Law, R.; Henchman, R.; McCammon, J. A gating mechanism proposed from a simulation of a human alpha7 nicotinic acetylcholine receptor. Proc. Natl. Acad. Sci. USA, 2005, 102, 6813-6818.

[18] Amiri, S.; Sansom, M.; Biggin, P. Molecular dynamics studies of AChBP with nicotine and carbamyl- choline: the role of water in the binding pocket. Protein Eng. Des. Sel., 2007, 20, 353-359.

[19] Bisson, W.; Westera, G.; Schubiger, P.; Scapozza, L. Homology modeling and dynamics of the extracellular domain of rat and human neuronal nicotinic acetylcholine receptor subtypes alpha4beta2 and alpha7. J. Mol. Model., 2008, 14, 891-899.

[20] Cheng, X.; Ivanov, I.; Wang, H.; Sine, S.; McCammon, J Nanosecond-timescale conformational dynamics of the human alpha7 nicotinic acetylcholine receptor. Biophys. J., 2007, 93, 2622-2634

[21] Wang, H.; Toghraee, R.; Papke, D.; Cheng, X.; McCammon, J.; Ravaioli, U.; Sine, S. Single-channel current through nicotinic receptor produced by closure of binding site C-loop. Biophys. J., 2009, 96, 3582-3590.

[22] Liu, X.; Xu, Y.; Wang, X.; Barrantes, F.; Jiang, H. Unbinding of nicotine from the acetylcholine binding protein: steered molecular dynamics simulations. J. Phys. Chem. B, 2008, 112, 4087-4893.

[23] Cheng, X.; Wang, H.; Grant, B.; Sine, S.; McCammon, J. Targeted molecular dynamics study of C-loop closure and channel gating in nicotinic receptors. PLoS Comput. Biol., 2006, 2, e134.

[24] Grazioso, G.; Cavalli, A.; Amici, M.D.; Recanatini, M.; Micheli, C.D. Alpha7 nicotinic acetylcholine re- ceptor agonists: prediction of their binding affinity through a molecular mechanics PoissonBoltzmann surface area approach. J. Comput. Chem., 2008, 29, 2593-2602.

[25] Huang, X.; Zheng, F.; Crooks, P.; Dwoskin, L.; Zhan, C. Modeling multiple species of nicotine and de- schloroepibatidine interacting with alpha4beta2 nicotinic acetylcholine receptor: from microscopic bind- ing to phenomenological binding affinity. J. Am. Chem. Soc., 2005, 127, 14401-14414.

[26] Huang, X.; Zheng, F.; Zhan, C. Modeling Differential Binding of alpha 4 beta 2 Nicotinic Acetylcholine Receptor with Agonists and Antagonists. J. Am. Chem. Soc., 2008, 130, 16691-16696.

[27] Schapira, M.; Abagyan, R.; Totrov, M. Structural model of nicotinic acetylcholine receptor isotypes bound to acetylcholine and nicotine. BMC Struct. Biol., 2002, 2, 1.

[28] Novère, N.L.; Grutter, T.; Changeux, J. Models of the extracellular domain of the nicotinic receptors and of agonist- and $\mathrm{Ca}^{2+}$-binding sites. Proc. Natl. Acad. Sci. USA, 2002, 99, 3210-3215.

[29] Grutter, T.; Novere, N.L.; Changeux, J. Rational understanding of nicotinic receptors drug binding. Curr. Top. Med. Chem., 2004, 4, $645-650$

[30] Babakhani, A.; Talley, T.; Taylor, P.; McCammon, J. A virtual screening study of the acetylcholine binding protein using a relaxed-complex approach. Comput. Biol. Chem., 2009, 33, 160170. 
[31] Gu, R.; Gu, H.; Xie, Z.; Wang, J.; Arias, H.; Wei, D.; Chou, K. Possible drug candidates for Alzheimer's disease deduced from studying their binding interactions with alpha7 nicotinic acetylcholine receptor. Med. Chem., 2009, 5, 250-262.

[32] Tasso, B.; Boido, C.C.; Terranova, E.; Gotti, C.; Riganti, L.; Clementi, F.; Artali, R.; Bombieri, G.; Meneghetti, F.; Sparatore, F. Synthesis, binding, and modeling studies of new cytisine derivatives, as ligands for neuronal nicotinic acetylcholine receptor subtypes. J. Med. Chem., 2009, 52, 4345-4357.

[33] Luttmann, E.; Ludwig, J.; Höffle-Maas, A.; Samochocki, M.; Maelicke, A.; Fels, G. Structural model for the binding sites of allosterically potentiating ligands on nicotinic acetylcholine receptors. ChemMedChem, 2009, 4, 1874-1882.

[34] Hu, Z.; Bai, L.; Tizabi, Y.; Southerland, W. Computational modeling study of human nicotinic acetylcholine receptor for developing new drugs in the treatment of alcoholism. Interdiscip. Sci., 2009, 1, 254-262.

[35] Parthiban, M.; Rajasekaran, M.; Ramakumar, S.; Shanmughavel, P. Molecular modeling of human pen- tameric alpha(7) neuronal nicotinic acetylcholine receptor and its interaction with its agonist and competitive antagonist. J. Biomol. Struct. Dyn., 2009, 26, 535547.

[36] Sander, T.; Bruun, A.; Balle, T. Docking to flexible nicotinic acetylcholine receptors: a validation study using the acetylcholine binding protein. J. Mol. Graph Model., 2010, 29, 415-424.

[37] Soriano, E.; Marco-Contelles, J.; Colmena, I.; Gandía, L. Computational analysis of the binding ability of heterocyclic and conformationally constrained epibatidine analogs in the neuronal nicotinic acetylcholine receptor. Mol. Divers., 2010, 14, 201-211.

[38] Cunningham, C.; Polston, J.; Jany, J.; Segert, I.; Miller, D. Interaction of lobeline and nicotinic receptor ligands with the discriminative stimulus properties of cocaine and amphetamine. Drug Alcohol. Depend., 2006, 84, 211-222.

[39] Farook, J.; Lewis, B.; Gaddis, J.; Littleton, J.; Barron, S. Lobeline, a nicotinic partial agonist attenuates alcohol consumption and preference in male C57BL/6J mice. Physiol. Behav., 2009, 97, 503506

[40] Zheng, G.; Dwoskin, L.; Deaciuc, A.; Norrholm, S.; Crooks, P. Defunctionalized lobeline analogues: structure-activity of novel ligands for the vesicular monoamine transporter. J. Med. Chem., 2005, 48, 5551-5560

[41] Crunelle, C.; Miller, M.; Booij, J.; van den Brink, W. The nicotinic acetylcholine receptor partial agonist varenicline and the treatment of drug dependence. Eur. Neuropsychopharmacol., 2010, 20, 6979.

[42] Locati, A.; Berthelot, M.; Evain, M.; Lebreton, J.; Questel, J.Y.L.; Mathé-Allainmat, M.; Planchat, A.; Renault, E.; Graton, J. The exceptional hydrogen-bond properties of neutral and protonated lobeline. J. Phys. Chem. A., 2007, 111, 6397-6405.

[43] Crooks, P.; Dwoskin, L. Use of lobeline epimers in the treatment of central nervous system diseases, pathologies, and drug abuse. WO Patent Application 2009/059260, 2009.

[44] Compere, D.; Marazano, C.; Das, B. Enantioselective access to Lobelia alkaloids. J. Org. Chem., 1999, 64, 4528-4532.

[45] Lape, R.; Colquhoun, D.; Sivilotti, L. On the nature of partial agonism in the nicotinic receptor superfamily. Nature, 2008, 454, 722-272.

[46] Astles, P.; Baker, S.; Boot, J.; Broad, L.; Dell, C.; Keenan, M. Recent progress in the development of subtype selective nicotinic acetylcholine receptor ligand. Curr. Drug Targets CNS Neurol. Disord., 2002, 1, 337-348.
[47] Jensen, A.; Frolund, B.; Liljefors, T.; Krogsgaard-Larsen, P Neuronal nicotinic acetylcholine receptors: structural revelations, target identifications, and therapeutic inspirations. J. Med. Chem., 2005, 48, 4705-4742.

[48] Gündisch, D.; Harms, K.; Schwarz, S.; Seitz, G.; Stubbs, M. Wegge, T. Synthesis and evaluation of diazine containing bioisosteres of ferruginine as ligands for nicotinic acetylcholine receptors. Bioorg. Med. Chem., 2001, 9, 2683-2691.

[49] Daly, J. Nicotinic agonists, antagonists, and modulators from natural sources. Cell Mol. Neurobiol., 2005, 25, 513-552.

[50] Miller, D.; Crooks, P.; Dwoskin, L. Lobeline inhibits nicotineevoked $[3 \mathrm{H}]$ dopamine overflow from rat striatal slices and nicotine-evoked ${ }^{86} \mathrm{Rb}+$ efflux from thalamic synaptosomes. Neuropharmacology, 2000, 39, 2654-2662.

[51] Briggs, C.; McKenna, D. Activation and inhibition of the human $\alpha 7$ nicotinic acetylcholine receptor by agonist binding affinity. Neuropharmacology, 1998, 37, 1095-1102.

[52] Edink, E.; Rucktooa, P.; Retra, K.; Akdemir, A.; Nahar, T.; Zuiderveld, O.; van Elk, R.; Janssen, E.; van Nierop, P.; van Muijlwijk-Koezen, J.; Smit, A.; Sixma, T.; Leurs, R.; de Esch, I. Fragment growing induces conformational changes in acetylcholine-binding protein: a structural and thermodynamic analysis. J. Am. Chem. Soc., 2011, 133, 5363-5371.

[53] Hansen, S.; Sulzenbacher, G.; Huxford, T.; Marchot, P.; Taylor, P.; Bourne Y. Structures of aplysia AChBP complexes with nicotinic agonists and antagonists reveal distinctive binding interfaces and conformations. EMBO J., 2005, 24, 2625-3646.

[54] O'Sullivan, O.; Suhre, K.; Abergel, C.; Higgins, D.; Notredame, C. 3DCoffee: combining protein sequences and structures within multiple sequence alignments. J. Mol. Biol., 2004, 340, 385-395.

[55] Hooft, R.; Vriend, G.; Sander, C.; Abola, E. Errors in protein structures. Nature, 1996, 381, 272-272.

[56] Ebnöther, A. Uber die mutarotation des lobelins. Cis-trans-isomere in der reihe des Lobelia-alkaloide. Helv. Chim. Acta, 1958, 41, 86396.

[57] Abagyan, R.; Totrov, M. High-throughput docking for lead generation. Curr. Opin. Chem. Biol., 2001, 5, 375-382.

[58] Mazur, A.; Abagyan, R. New methodology for computer-aided modelling of biomolecular structure and dynamics. 1. Non-cyclic structures. J. Biomol. Struct. Dyn., 1989, 6, 815-132.

[59] Abagyan, R.; Mazur, A. New methodology for computer-aided modelling of biomolecular structure and dynamics. 2. Loca deformations and cycles. J. Biomol. Struct. Dyn., 1989, 6, 833-845.

[60] Totrov, M.; Abagyan, R. Derivation of sensitive discimination potiential for virtual ligand screening. Proceeding of the Third Annual Intl. Conf. Comp. Mol. Biol, 1999. April 11 - 14, 1999 Lyon, France, 312

[61] Glaser, R.; Hug, P.; Drouin, M.; Michel, A. Solution- and solidstate stereochemistry of (-)- $\alpha$-lobeline hydrobromide and hydrochloride, a respiratory-stimulant drug. J. Chem. Soc. Perkin Trans., 1992, 2, 1071.

[62] Barlow, R.; Johnson, O. Relations between structure and nicotinelike activity: X-ray crystal structure analysis of ()- cytisine and ()lobeline hydrochloride and a comparison with ()-nicotine and other nicotine- like compounds. Br. J. Pharmacol., 1989, 98, 799-808.

[63] Sgrignani, J.; Bonaccini, C.; Grazioso, G.; Chioccioli, M.; Cavalli, A.; Grateri, P. Insights into docking and scoring neuronal a4b2 nicotinic receptor agonists using molecular dynamics simulations and QM/MM calculations. J. Comput. Chem., 2009, 30, 2443-2454. 